Скопје, Македонија

\title{
SOME NEW OSTROWSKI TYPE INEQUALITIES FOR GENERALIZED $(s, m, \varphi)$-PREINVEX FUNCTIONS VIA FRACTIONAL INTEGRAL OPERATORS
}

\author{
ARTION KASHURI AND ROZANA LIKO
}

\begin{abstract}
In the present paper, the notion of generalized $(s, m, \varphi)$-preinvex function is applied to establish some new generalizations of Ostrowski type inequalities via fractional integral operators. These results not only extend the results appeared in the literature (see [1]) but also provide new estimates on these type. Some applications to special means are also given.
\end{abstract}

\section{InTRoduction AND PRELIMINARIES}

The following notations are used throughout this paper. We use $I$ to denote an interval on the real line $\mathbb{R}=(-\infty,+\infty)$ and $I^{\circ}$ to denote the interior of $I$. For any subset $K \subseteq \mathbb{R}^{n}, K^{\circ}$ is used to denote the interior of $K$. $\mathbb{R}^{n}$ is used to denote a $n$-dimensional vector space. The set of integrable functions on the interval $[a, b]$ is denoted by $L_{1}[a, b]$.

The following result is known in the literature as the Ostrowski inequality (see [37]), which gives an upper bound for the approximation of the integral average $\frac{1}{b-a} \int_{a}^{b} f(t) d t$ by the value $f(x)$ at point $x \in[a, b]$.

Theorem 1. Let $f: I \longrightarrow \mathbb{R}$ be a mapping differentiable in $I^{\circ}$ and let $a, b \in I^{\circ}$ with $a<b$. If $\left|f^{\prime}(x)\right| \leq M$ for all $x \in[a, b]$, then

$$
\left|f(x)-\frac{1}{b-a} \int_{a}^{b} f(t) d t\right| \leq M(b-a)\left[\frac{1}{4}+\frac{\left(x-\frac{a+b}{2}\right)^{2}}{(b-a)^{2}}\right], \quad \forall x \in[a, b] .
$$

For other recent results concerning Ostrowski type inequalities (see [28]-[32],[37], [38]). Ostrowski inequality is playing a very important role in all the fields of mathematics, especially in the theory of approximations. Thus such inequalities were studied extensively by many researches and numerous generalizations, extensions and variants of them for various kind of functions like bounded variation,

2000 Mathematics Subject Classification. Primary: 26A51; Secondary: 26A33, 26D07, 26D10, 26D15.

Key words and phrases. Ostrowski type inequality, Hölder's inequality, power mean inequality, Riemann-Liouville fractional integral, fractional integral operator, $s$-convex function in the second sense, $m$-invex. 
synchronous, Lipschitzian, monotonic, absolutely, continuous and $n$-times differentiable mappings etc. appeared in a number of papers (see [3]-[8],[10]-[13]). In recent years, one more dimension has been added to this studies, by introducing a number of integral inequalities involving various fractional operators like RiemannLiouville, Erdelyi-Kober, Katugampola, conformable fractional integral operators etc. by many authors (see [14]-[25]). Riemann-Liouville fractional integral operators are the most central between these fractional operators.

Fractional calculus (see [36]), was introduced at the end of the nineteenth century by Liouville and Riemann, the subject of which has become a rapidly growing area and has found applications in diverse fields ranging from physical sciences and engineering to biological sciences and economics.

Definition 1. Let $f \in L_{1}[a, b]$. The Riemann-Liouville integrals $J_{a+}^{\alpha} f$ and $J_{b-}^{\alpha} f$ of order $\alpha>0$ with $a \geq 0$ are defined by

$$
J_{a+}^{\alpha} f(x)=\frac{1}{\Gamma(\alpha)} \int_{a}^{x}(x-t)^{\alpha-1} f(t) d t, \quad x>a
$$

and

$$
J_{b-}^{\alpha} f(x)=\frac{1}{\Gamma(\alpha)} \int_{x}^{b}(t-x)^{\alpha-1} f(t) d t, \quad b>x,
$$

where $\Gamma(\alpha)=\int_{0}^{+\infty} e^{-u} u^{\alpha-1} d u$. Here $J_{a+}^{0} f(x)=J_{b-}^{0} f(x)=f(x)$.

In the case of $\alpha=1$, the fractional integral reduces to the classical integral.

Due to the wide application of fractional integrals, some authors extended to study fractional Ostrowski type inequalities for functions of different classes (see $[36])$.

In (see [23]), Raina introduced a class of functions defined formally by

$$
\mathcal{F}_{\rho, \lambda}^{\sigma}(x)=\mathcal{F}_{\rho, \lambda}^{\sigma(0), \sigma(1), \ldots}(x)=\sum_{k=0}^{+\infty} \frac{\sigma(k)}{\Gamma(\rho k+\lambda)} x^{k} \quad(\rho, \lambda>0 ;|x|<\mathbb{R}),
$$

where the coefficients $(\sigma(k), k \in \mathbb{N} \cup\{0\})$ is a bounded sequence of positive real numbers. With the help of (1.2), Raina (see [23]) and Agarwal et al. (see [4]) defined the following left-sided and right-sided fractional integral operators respectively, as follows:

$$
\begin{array}{ll}
\left(\mathcal{J}_{\rho, \lambda, a+; w}^{\sigma} \varphi\right)(x)=\int_{a}^{x}(x-t)^{\lambda-1} \mathcal{F}_{\rho, \lambda}^{\sigma}\left[w(x-t)^{\rho}\right] \varphi(t) d t & (x>a>0), \\
\left(\mathcal{J}_{\rho, \lambda, b-; w}^{\sigma} \varphi\right)(x)=\int_{x}^{b}(t-x)^{\lambda-1} \mathcal{F}_{\rho, \lambda}^{\sigma}\left[w(t-x)^{\rho}\right] \varphi(t) d t & (0<x<b),
\end{array}
$$

where $\lambda, \rho>0, w \in \mathbb{R}$ and $\varphi(t)$ is such that the integral on the right side exits. It is easy to verify that $\mathcal{J}_{\rho, \lambda, a+; w}^{\sigma} \varphi(x)$ and $\mathcal{J}_{\rho, \lambda, b-; w}^{\sigma} \varphi(x)$ are bounded integral operators on $L_{1}(a, b)$, if

$$
\mathfrak{R}:=\mathcal{F}_{\rho, \lambda+1}^{\sigma}\left[w(b-a)^{\rho}\right]<\infty .
$$

In fact, for $\varphi \in L_{1}(a, b)$, we have

$$
\left\|\mathcal{J}_{\rho, \lambda, a+; w}^{\sigma} \varphi(x)\right\|_{1} \leq \mathfrak{R}(b-a)^{\lambda}\|\varphi\|_{1}
$$


and

where

$$
\left\|\mathcal{J}_{\rho, \lambda, b-; w}^{\sigma} \varphi(x)\right\|_{1} \leq \mathfrak{R}(b-a)^{\lambda}\|\varphi\|_{1}
$$

$$
\|\varphi\|_{p}:=\left(\int_{a}^{b}|\varphi(t)|^{p} d t\right)^{\frac{1}{p}} .
$$

The importance of these operators stems indeed from their generality. Many useful fractional integral operators can be obtained by specializing the coefficient $\sigma(k)$. For instance the classical Riemann-Liouville fractional integrals $J_{a+}^{\alpha}$ and $J_{b-}^{\alpha}$ of order $\alpha$ follow easily by setting $\lambda=\alpha, \sigma(0)=1$ and $w=0$ in (1.3) and (1.4). Now, let us evoke some definitions.

Definition 2. (see [27]) A function $f:[0,+\infty) \longrightarrow \mathbb{R}$ is said to be s-convex in the second sense, if

$$
f(\lambda x+(1-\lambda) y) \leq \lambda^{s} f(x)+(1-\lambda)^{s} f(y)
$$

for all $x, y \geq 0, \lambda \in[0,1]$ and $s \in(0,1]$.

It is clear that a 1 -convex function must be convex on $[0,+\infty)$ as usual. The $s$-convex functions in the second sense have been investigated in (see [27]).

Definition 3. (see [33]) $A$ set $K \subseteq \mathbb{R}^{n}$ is said to be invex with respect to the mapping $\eta: K \times K \longrightarrow \mathbb{R}^{n}$, if $x+t \eta(y, x) \in K$ for every $x, y \in K$ and $t \in[0,1]$.

Notice that every convex set is invex with respect to the mapping $\eta(y, x)=y-x$, but the converse is not necessarily true. For more details (see [33], [34]).

Definition 4. (see [35]) The function $f$ defined on the invex set $K \subseteq \mathbb{R}^{n}$ is said to be preinvex with respect $\eta$, if for every $x, y \in K$ and $t \in[0,1]$, we have that

$$
f(x+t \eta(y, x)) \leq(1-t) f(x)+t f(y) .
$$

The concept of preinvexity is more general than convexity since every convex function is preinvex with respect to the mapping $\eta(y, x)=y-x$, but the converse is not true.

The aim of this paper is to establish some generalizations of Ostrowski type inequalities using new identity given in Section 2 for generalized $(s, m, \varphi)$-preinvex functions via generalized fractional integral operators. In Section 3, some applications to special means are given. In Section 4, some conclusions and future research are given. These results not only extend the results appeared in the literature (see [1]) but also provide new estimates on these type.

\section{MAIN RESULTS}

Definition 5. (see [26]) $A$ set $K \subseteq \mathbb{R}^{n}$ is said to be $m$-invex with respect to the mapping $\eta: K \times K \times(0,1] \longrightarrow \mathbb{R}^{n}$ for some fixed $m \in(0,1]$, if $m x+t \eta(y, x, m) \in K$ holds for each $x, y \in K$ and any $t \in[0,1]$.

Remark 1. In Definition 5, under certain conditions, the mapping $\eta(y, x, m)$ could reduce to $\eta(y, x)$. For example when $m=1$, then the $m$-invex set degenerates an invex set on $K$. 
Definition 6. (see [2]) Let $K \subseteq \mathbb{R}$ be an open m-invex set with respect to $\eta$ : $K \times K \times(0,1] \longrightarrow \mathbb{R}$ and $\varphi: I \longrightarrow K$ a continuous function. For $f: K \longrightarrow \mathbb{R}$ and any fixed $s, m \in(0,1]$, if

$$
f(m \varphi(x)+t \eta(\varphi(y), \varphi(x), m)) \leq m(1-t)^{s} f(\varphi(x))+t^{s} f(\varphi(y))
$$

is valid for all $x, y \in I, t \in[0,1]$, then we say that $f(x)$ is generalized $(s, m, \varphi)$ preinvex function with respect to $\eta$.

Remark 2. In Definition 6, it is worthwhile to note that the class of generalized $(s, m, \varphi)$-preinvex function is a generalization of the class of $s$-convex in the second sense function given in Definition 2.

Throughout this paper we denote

$$
\begin{gathered}
I_{f, \eta, \varphi}(x ; \lambda, \rho, w, m, a, b) \\
=\left[\frac{(x-m \varphi(a))^{\lambda} \mathcal{F}_{\rho, \lambda+1}^{\sigma}\left[w(x-m \varphi(a))^{\rho}\right]}{\eta^{\lambda+1}(\varphi(b), \varphi(a), m)}\right. \\
\left.+\frac{(m \varphi(a)+\eta(\varphi(b), \varphi(a), m)-x)^{\lambda} \mathcal{F}_{\rho, \lambda+1}^{\sigma}\left[w(m \varphi(a)+\eta(\varphi(b), \varphi(a), m)-x)^{\rho}\right]}{\eta^{\lambda+1}(\varphi(b), \varphi(a), m)}\right] f(x) \\
-\frac{1}{\eta^{\lambda+1}(\varphi(b), \varphi(a), m)} \\
\times\left[\left(\mathcal{J}_{\rho, \lambda, x-; w}^{\sigma} f\right)(m \varphi(a))+\left(\mathcal{J}_{\rho, \lambda, x+; w}^{\sigma} f\right)(m \varphi(a)+\eta(\varphi(b), \varphi(a), m))\right] .
\end{gathered}
$$

In this section, in order to prove our main results regarding some generalizations of Ostrowski type inequalities for generalized $(s, m, \varphi)$-preinvex functions via generalized fractional integral operators, we need the following new interesting Lemma:

Lemma 1. Let $\varphi: I \longrightarrow K$ be a continuous function. Suppose $K \subseteq \mathbb{R}$ be an open $m$-invex subset with respect to $\eta: K \times K \times(0,1] \longrightarrow \mathbb{R}$ for any fixed $m \in(0,1]$ and let $\eta(\varphi(b), \varphi(a), m) \neq 0$. Assume that $f: K \longrightarrow \mathbb{R}$ is a differentiable function on $K^{\circ}$. If $f^{\prime} \in L_{1}[m \varphi(a), m \varphi(a)+\eta(\varphi(b), \varphi(a), m)]$, then we have the following identity involving generalized fractional integral operators:

$$
I_{f, \eta, \varphi}(x ; \lambda, \rho, w, m, a, b)=\int_{0}^{1} \theta(t) f^{\prime}(m \varphi(a)+t \eta(\varphi(b), \varphi(a), m)) d t
$$

for each $t \in[0,1]$, where $\lambda, \rho>0, w \in \mathbb{R}$ and

$$
\theta(t)= \begin{cases}t^{\lambda} \mathcal{F}_{\rho, \lambda+1}^{\sigma}\left[w \eta^{\rho}(\varphi(b), \varphi(a), m) t^{\rho}\right], & t \in\left[0, \frac{x-m \varphi(a)}{\eta(\varphi(b), \varphi(a), m)}\right) ; \\ (1-t)^{\lambda} \mathcal{F}_{\rho, \lambda+1}^{\sigma}\left[w \eta^{\rho}(\varphi(b), \varphi(a), m)(1-t)^{\rho}\right], & t \in\left[\frac{x-m \varphi(a)}{\eta(\varphi(b), \varphi(a), m)}, 1\right) .\end{cases}
$$

Proof. Integrating by parts, we get

$$
\begin{gathered}
\int_{0}^{1} \theta(t) f^{\prime}(m \varphi(a)+t \eta(\varphi(b), \varphi(a), m)) d t \\
=\int_{0}^{\frac{x-m \varphi(a)}{\eta(\varphi(b), \varphi(a), m)}} t^{\lambda} \mathcal{F}_{\rho, \lambda+1}^{\sigma}\left[w \eta^{\rho}(\varphi(b), \varphi(a), m) t^{\rho}\right] f^{\prime}(m \varphi(a)+t \eta(\varphi(b), \varphi(a), m)) d t
\end{gathered}
$$




$$
\begin{gathered}
+\int_{\frac{x-m \varphi(a)}{\eta(\varphi(b), \varphi(a), m)}}^{1}(1-t)^{\lambda} \mathcal{F}_{\rho, \lambda+1}^{\sigma}\left[w \eta^{\rho}(\varphi(b), \varphi(a), m)(1-t)^{\rho}\right] \\
\times f^{\prime}(m \varphi(a)+t \eta(\varphi(b), \varphi(a), m)) d t \\
=\left.t^{\lambda} \mathcal{F}_{\rho, \lambda+1}^{\sigma}\left[w \eta^{\rho}(\varphi(b), \varphi(a), m) t^{\rho}\right] \frac{f(m \varphi(a)+t \eta(\varphi(b), \varphi(a), m))}{\eta(\varphi(b), \varphi(a), m)}\right|_{0} ^{\frac{x-m \varphi(a)}{\eta(\varphi(b), \varphi(a), m)}} \\
-\int_{0}^{\frac{x-m \varphi(a)}{\eta(\varphi(b), \varphi(a), m)}} t^{\lambda-1} \mathcal{F}_{\rho, \lambda}^{\sigma}\left[w \eta^{\rho}(\varphi(b), \varphi(a), m) t^{\rho}\right] \frac{f(m \varphi(a)+t \eta(\varphi(b), \varphi(a), m))}{\eta(\varphi(b), \varphi(a), m)} d t \\
+\left.(1-t)^{\lambda} \mathcal{F}_{\rho, \lambda+1}^{\sigma}\left[w \eta^{\rho}(\varphi(b), \varphi(a), m)(1-t)^{\rho}\right] \frac{f(m \varphi(a)+t \eta(\varphi(b), \varphi(a), m))}{\eta(\varphi(b), \varphi(a), m)}\right|_{\frac{x-m \varphi(a)}{\eta(\varphi(b), \varphi(a), m)}} ^{1} \\
-\int_{\frac{x-m \varphi(a)}{\eta(\varphi(b), \varphi(a), m)}}(1-t)^{\lambda-1} \mathcal{F}_{\rho, \lambda}^{\sigma}\left[w \eta^{\rho}(\varphi(b), \varphi(a), m)(1-t)^{\rho}\right] \\
\times \frac{f(m \varphi(a)+t \eta(\varphi(b), \varphi(a), m))}{\eta(\varphi(b), \varphi(a), m)} d t \\
=\left[\frac{(x-m \varphi(a))^{\lambda} \mathcal{F}_{\rho, \lambda+1}^{\sigma}\left[w(x-m \varphi(a))^{\rho}\right]}{\eta^{\lambda+1}(\varphi(b), \varphi(a), m)}\right. \\
\left.+\frac{\left.(m \varphi(a)+\eta(\varphi(b), \varphi(a), m)-x)^{\lambda} \mathcal{F}_{\rho, \lambda+1}^{\sigma}\left[w(m \varphi(a)+\eta(\varphi(b), \varphi(a), m)-x)^{\rho}\right]\right]}{\eta^{\lambda+1}(\varphi(b), \varphi(a), m)}\right] f(x) \\
-\frac{1}{\eta^{\lambda+1}(\varphi(b), \varphi(a), m)} \\
\times\left[\left(\mathcal{J}_{\rho, \lambda, x-; w}^{\sigma} f\right)(m \varphi(a))+\left(\mathcal{J}_{\rho, \lambda, x+; w}^{\sigma} f\right)(m \varphi(a)+\eta(\varphi(b), \varphi(a), m))\right] .
\end{gathered}
$$

By using Lemma 1, one can extend to the following results.

Theorem 2. Let $\varphi: I \longrightarrow A$ be a continuous function. Suppose $A \subseteq \mathbb{R}$ be an open $m$-invex subset with respect to $\eta: A \times A \times(0,1] \longrightarrow \mathbb{R}$ for any fixed $s, m \in(0,1]$ and let $\eta(\varphi(b), \varphi(a), m) \neq 0$. Assume that $f: A \longrightarrow \mathbb{R}$ is a differentiable function on $A^{\circ}$. If $\left|f^{\prime}\right|$ is generalized $(s, m, \varphi)$-preinvex function on $[m \varphi(a), m \varphi(a)+\eta(\varphi(b), \varphi(a), m)]$, then the following inequality for generalized fractional integral operators holds:

$$
\begin{gathered}
\left|I_{f, \eta, \varphi}(x ; \lambda, \rho, w, m, a, b)\right| \\
\leq m\left[\frac{(m \varphi(a)+\eta(\varphi(b), \varphi(a), m)-x)^{\lambda+s+1}}{\eta^{\lambda+s+1}(\varphi(b), \varphi(a), m)} \mathcal{F}_{\rho, \lambda+1}^{\sigma_{2}}\left[|w|(m \varphi(a)+\eta(\varphi(b), \varphi(a), m)-x)^{\rho}\right]\right. \\
\left.+\mathcal{F}_{\rho, \lambda+1}^{\sigma_{1}}\left[|w| \eta^{\rho}(\varphi(b), \varphi(a), m)\right]\right]\left|f^{\prime}(\varphi(a))\right| \\
+\left[\frac{(x-m \varphi(a))^{\lambda+s+1}}{\eta^{\lambda+s+1}(\varphi(b), \varphi(a), m)} \mathcal{F}_{\rho, \lambda+1}^{\sigma_{2}}\left[|w|(x-m \varphi(a))^{\rho}\right]\right.
\end{gathered}
$$




$$
\left.+\mathcal{F}_{\rho, \lambda+1}^{\sigma_{3}}\left[|w| \eta^{\rho}(\varphi(b), \varphi(a), m)\right]\right]\left|f^{\prime}(\varphi(b))\right|,
$$

where $\lambda, \rho>0, w \in \mathbb{R}, k=0,1,2, \ldots, \beta(x ; a, b)$ is incompleted beta function and

$$
\begin{gathered}
\sigma_{1}(k)=\sigma(k) \beta\left(\frac{x-m \varphi(a)}{\eta(\varphi(b), \varphi(a), m)} ; \lambda+\rho k+1, s+1\right) ; \\
\sigma_{2}(k)=\sigma(k) \frac{1}{\lambda+\rho k+s+1} ; \\
\sigma_{3}(k)=\sigma(k) \beta\left(\frac{m \varphi(a)+\eta(\varphi(b), \varphi(a), m)-x}{\eta(\varphi(b), \varphi(a), m)} ; \lambda+\rho k+1, s+1\right) .
\end{gathered}
$$

Proof. From Lemma 1, generalized $(s, m, \varphi)$-preinvexity of $\left|f^{\prime}\right|$ and properties of the modulus, we have

$$
\begin{gathered}
\quad\left|I_{f, \eta, \varphi}(x ; \lambda, \rho, w, m, a, b)\right| \\
\leq \int_{0}^{\frac{x-m \varphi(a)}{\eta(\varphi(b), \varphi(a), m)}} t^{\lambda} \mathcal{F}_{\rho, \lambda+1}^{\sigma}\left[|w| \eta^{\rho}(\varphi(b), \varphi(a), m) t^{\rho}\right]\left|f^{\prime}(m \varphi(a)+t \eta(\varphi(b), \varphi(a), m))\right| d t \\
+\int_{\frac{x-m \varphi(a)}{\eta(\varphi(b), \varphi(a), m)}}^{1}|1-t|^{\lambda} \mathcal{F}_{\rho, \lambda+1}^{\sigma}\left[|w| \eta^{\rho}(\varphi(b), \varphi(a), m)(1-t)^{\rho}\right] \\
\quad \times\left|f^{\prime}(m \varphi(a)+t \eta(\varphi(b), \varphi(a), m))\right| d t \\
\leq \int_{0}^{\frac{x-m \varphi(a)}{\eta(\varphi(b), \varphi(a), m)}} t^{\lambda} \mathcal{F}_{\rho, \lambda+1}^{\sigma}\left[|w| \eta^{\rho}(\varphi(b), \varphi(a), m) t^{\rho}\right]\left[m(1-t)^{s}\left|f^{\prime}(\varphi(a))\right|+t^{s}\left|f^{\prime}(\varphi(b))\right|\right] d t \\
+\int_{\frac{x-m \varphi(a)}{\eta(\varphi(b), \varphi(a), m)}}^{1}(1-t)^{\lambda} \mathcal{F}_{\rho, \lambda+1}^{\sigma}\left[|w| \eta^{\rho}(\varphi(b), \varphi(a), m)(1-t)^{\rho}\right] \\
\times\left[m(1-t)^{s}\left|f^{\prime}(\varphi(a))\right|+t^{s}\left|f^{\prime}(\varphi(b))\right|\right] d t \\
=m\left[\frac{(m \varphi(a)+\eta(\varphi(b), \varphi(a), m)-x)^{\lambda+s+1}}{\eta^{\lambda+s+1}(\varphi(b), \varphi(a), m)} \mathcal{F}_{\rho, \lambda+1}^{\sigma_{2}}\left[|w|(m \varphi(a)+\eta(\varphi(b), \varphi(a), m)-x)^{\rho}\right]\right. \\
+\mathcal{F}_{\rho, \lambda+1}^{\sigma_{1}}\left[|w| \eta^{\rho}(\varphi(b), \varphi(a), m)\right]\left|f^{\prime}(\varphi(a))\right| \\
+\left[\begin{array}{c}
(x-m \varphi(a))^{\lambda+s+1} \\
\eta^{\lambda+s+1}(\varphi(b), \varphi(a), m)
\end{array}\right. \\
+\mathcal{F}_{\rho, \lambda+1}^{\sigma_{2}}\left[|w|(x-m \varphi(a))^{\rho}\right] \\
\mathcal{F}_{\rho, \lambda+1}^{\sigma_{3}}\left[|w| \eta^{\rho}(\varphi(b), \varphi(a), m)\right]\left|f^{\prime}(\varphi(b))\right| .
\end{gathered}
$$

Corollary 1. Under the same conditions as in Theorem 2, if we choose $m=s=$ $1, \eta(\varphi(b), \varphi(a), m)=\varphi(b)-m \varphi(a)$ and $\varphi(x)=x$, we get

$$
\begin{gathered}
\mid\left[\frac{(x-a)^{\lambda} \mathcal{F}_{\rho, \lambda+1}^{\sigma}\left[w(x-a)^{\rho}\right]+(b-x)^{\lambda} \mathcal{F}_{\rho, \lambda+1}^{\sigma}\left[w(b-x)^{\rho}\right]}{(b-a)^{\lambda+1}}\right] f(x) \\
\quad-\frac{1}{(b-a)^{\lambda+1}}\left[\left(\mathcal{J}_{\rho, \lambda, x-; w}^{\sigma} f\right)(a)+\left(\mathcal{J}_{\rho, \lambda, x+; w}^{\sigma} f\right)(b)\right] \mid
\end{gathered}
$$




$$
\begin{gathered}
\leq\left[\frac{(b-x)^{\lambda+2}}{(b-a)^{\lambda+2}} \mathcal{F}_{\rho, \lambda+1}^{\sigma_{2}^{*}}\left[|w|(b-x)^{\rho}\right]+\mathcal{F}_{\rho, \lambda+1}^{\sigma_{1}^{*}}\left[|w|(b-a)^{\rho}\right]\right]\left|f^{\prime}(a)\right| \\
+\left[\frac{(x-a)^{\lambda+2}}{(b-a)^{\lambda+2}} \mathcal{F}_{\rho, \lambda+1}^{\sigma_{2}^{*}}\left[|w|(x-a)^{\rho}\right]+\mathcal{F}_{\rho, \lambda+1}^{\sigma_{3}^{*}}\left[|w|(b-a)^{\rho}\right]\right]\left|f^{\prime}(b)\right|,
\end{gathered}
$$

where

$$
\begin{gathered}
\sigma_{1}^{*}(k)=\sigma(k) \beta\left(\frac{x-a}{b-a} ; \lambda+\rho k+1,2\right) ; \quad \sigma_{2}^{*}(k)=\sigma(k) \frac{1}{\lambda+\rho k+2} ; \\
\sigma_{3}^{*}(k)=\sigma(k) \beta\left(\frac{b-x}{b-a} ; \lambda+\rho k+1,2\right) .
\end{gathered}
$$

Corollary 2. If we choose $\sigma(0)=1, w=0$ in Corollary 1, the inequality (2.5) reduces to inequality (2.1) of (see [1] Theorem 2.1).

Theorem 3. Let $\varphi: I \longrightarrow A$ be a continuous function. Suppose $A \subseteq \mathbb{R}$ be an open $m$-invex subset with respect to $\eta: A \times A \times(0,1] \longrightarrow \mathbb{R}$ for any fixed $s, m \in(0,1]$ and let $\eta(\varphi(b), \varphi(a), m) \neq 0$. Assume that $f: A \longrightarrow \mathbb{R}$ is a differentiable function on $A^{\circ}$. If $\left|f^{\prime}\right|^{q}$ is generalized $(s, m, \varphi)$-preinvex function on $[m \varphi(a), m \varphi(a)+\eta(\varphi(b), \varphi(a), m)], q>1, p^{-1}+q^{-1}=1$, then the following inequality for generalized fractional integral operators holds:

$$
\begin{gathered}
\left|I_{f, \eta, \varphi}(x ; \lambda, \rho, w, m, a, b)\right| \leq \frac{1}{(s+1)^{\frac{1}{q}}} \frac{1}{\eta^{\lambda+\frac{s}{q}+1}(\varphi(b), \varphi(a), m)} \\
\times\left\{\left[m\left[\eta^{s+1}(\varphi(b), \varphi(a), m)-(m \varphi(a)+\eta(\varphi(b), \varphi(a), m)-x)^{s+1}\right]\left|f^{\prime}(\varphi(a))\right|^{q}\right.\right. \\
\left.+(x-m \varphi(a))^{s+1}\left|f^{\prime}(\varphi(b))\right|^{q}\right]^{\frac{1}{q}}(x-m \varphi(a))^{\lambda+\frac{1}{p}} \mathcal{F}_{\rho, \lambda+1}^{\sigma^{*}}\left[|w|(x-m \varphi(a))^{\rho}\right] \\
+\left[m\left[(m \varphi(a)+\eta(\varphi(b), \varphi(a), m)-x)^{s+1}\right]\left|f^{\prime}(\varphi(a))\right|^{q}\right. \\
\left.+\left[\eta^{s+1}(\varphi(b), \varphi(a), m)-(x-m \varphi(a))^{s+1}\right]\left|f^{\prime}(\varphi(b))\right|^{q}\right]^{\frac{1}{q}} \\
\left.\times(m \varphi(a)+\eta(\varphi(b), \varphi(a), m)-x)^{\lambda+\frac{1}{p}} \mathcal{F}_{\rho, \lambda+1}^{\sigma^{*}}\left[|w|(m \varphi(a)+\eta(\varphi(b), \varphi(a), m)-x)^{\rho}\right]\right\},
\end{gathered}
$$

where $\lambda, \rho>0, w \in \mathbb{R}, k=0,1,2, \ldots$ and

$$
\sigma^{*}(k)=\sigma(k)\left(\frac{1}{(\lambda+\rho k) p+1}\right)^{\frac{1}{p}}
$$

Proof. Suppose that $q>1$. From Lemma 1, generalized $(s, m, \varphi)$-preinvexity of $\left|f^{\prime}\right|^{q}$, Hölder inequality and properties of the modulus, we have

$$
\begin{gathered}
\left|I_{f, \eta, \varphi}(x ; \lambda, \rho, w, m, a, b)\right| \\
\leq \int_{0}^{\frac{x-m \varphi(a)}{\eta(\varphi(b), \varphi(a), m)}} t^{\lambda} \mathcal{F}_{\rho, \lambda+1}^{\sigma}\left[|w| \eta^{\rho}(\varphi(b), \varphi(a), m) t^{\rho}\right]\left|f^{\prime}(m \varphi(a)+t \eta(\varphi(b), \varphi(a), m))\right| d t \\
+\int_{\frac{x-m \varphi(a)}{\eta(\varphi(b), \varphi(a), m)}}^{1}|1-t|^{\lambda} \mathcal{F}_{\rho, \lambda+1}^{\sigma}\left[|w| \eta^{\rho}(\varphi(b), \varphi(a), m)(1-t)^{\rho}\right]
\end{gathered}
$$


SOME NEW OSTROWSKI TYPE INEQUALITIES...

$$
\begin{aligned}
& \times\left|f^{\prime}(m \varphi(a)+t \eta(\varphi(b), \varphi(a), m))\right| d t \\
& \leq \sum_{k=0}^{+\infty} \frac{\sigma(k)|w|^{k} \eta^{\rho k}(\varphi(b), \varphi(a), m)}{\Gamma(\lambda+\rho k+1)} \times\left\{\left(\int_{0}^{\frac{x-m \varphi(a)}{\eta(\varphi(b), \varphi(a), m)}} t^{(\lambda+\rho k) p} d t\right)^{\frac{1}{p}}\right. \\
& \times\left(\int_{0}^{\frac{x-m \varphi(a)}{\eta(\varphi(b), \varphi(a), m)}}\left|f^{\prime}(m \varphi(a)+t \eta(\varphi(b), \varphi(a), m))\right|^{q} d t\right)^{\frac{1}{q}} \\
& +\left(\int_{\frac{x-m \varphi(a)}{\eta(\varphi(b), \varphi(a), m)}}^{1}(1-t)^{(\lambda+\rho k) p} d t\right)^{\frac{1}{p}} \\
& \left.\times\left(\int_{\frac{x-m \varphi(a)}{\eta(\varphi(b), \varphi(a), m)}}^{1}\left|f^{\prime}(m \varphi(a)+t \eta(\varphi(b), \varphi(a), m))\right|^{q} d t\right)^{\frac{1}{q}}\right\} \\
& \leq \sum_{k=0}^{+\infty} \frac{\sigma(k)|w|^{k} \eta^{\rho k}(\varphi(b), \varphi(a), m)}{\Gamma(\lambda+\rho k+1)} \times\left\{\left(\int_{0}^{\frac{x-m \varphi(a)}{\eta(\varphi(b), \varphi(a), m)}} t^{(\lambda+\rho k) p} d t\right)^{\frac{1}{p}}\right. \\
& \times\left(\int_{0}^{\frac{x-m \varphi(a)}{\eta(\varphi(b), \varphi(a), m)}}\left[m(1-t)^{s}\left|f^{\prime}(\varphi(a))\right|^{q}+t^{s}\left|f^{\prime}(\varphi(b))\right|^{q}\right] d t\right)^{\frac{1}{q}} \\
& +\left(\int_{\frac{x-m \varphi(a)}{\eta(\varphi(b), \varphi(a), m)}}^{1}(1-t)^{(\lambda+\rho k) p} d t\right)^{\frac{1}{p}} \\
& \left.\times\left(\int_{\frac{x-m \varphi(a)}{\eta(\varphi(b), \varphi(a), m)}}^{1}\left[m(1-t)^{s}\left|f^{\prime}(\varphi(a))\right|^{q}+t^{s}\left|f^{\prime}(\varphi(b))\right|^{q}\right] d t\right)^{\frac{1}{q}}\right\} \\
& =\frac{1}{(s+1)^{\frac{1}{q}}} \frac{1}{\eta^{\lambda+\frac{s}{q}+1}(\varphi(b), \varphi(a), m)} \\
& \times\left\{\left[m\left[\eta^{s+1}(\varphi(b), \varphi(a), m)-(m \varphi(a)+\eta(\varphi(b), \varphi(a), m)-x)^{s+1}\right]\left|f^{\prime}(\varphi(a))\right|^{q}\right.\right. \\
& \left.+(x-m \varphi(a))^{s+1}\left|f^{\prime}(\varphi(b))\right|^{q}\right]^{\frac{1}{q}}(x-m \varphi(a))^{\lambda+\frac{1}{p}} \mathcal{F}_{\rho, \lambda+1}^{\sigma^{*}}\left[|w|(x-m \varphi(a))^{\rho}\right] \\
& +\left[m\left[(m \varphi(a)+\eta(\varphi(b), \varphi(a), m)-x)^{s+1}\right]\left|f^{\prime}(\varphi(a))\right|^{q}\right. \\
& \left.+\left[\eta^{s+1}(\varphi(b), \varphi(a), m)-(x-m \varphi(a))^{s+1}\right]\left|f^{\prime}(\varphi(b))\right|^{q}\right]^{\frac{1}{q}} \\
& \left.\times(m \varphi(a)+\eta(\varphi(b), \varphi(a), m)-x)^{\lambda+\frac{1}{p}} \mathcal{F}_{\rho, \lambda+1}^{\sigma^{*}}\left[|w|(m \varphi(a)+\eta(\varphi(b), \varphi(a), m)-x)^{\rho}\right]\right\} .
\end{aligned}
$$


Corollary 3. Under the same conditions as in Theorem 3, if we choose $m=s=$ $1, \eta(\varphi(b), \varphi(a), m)=\varphi(b)-m \varphi(a)$ and $\varphi(x)=x$, we get

$$
\begin{gathered}
\mid\left[\frac{(x-a)^{\lambda} \mathcal{F}_{\rho, \lambda+1}^{\sigma}\left[w(x-a)^{\rho}\right]+(b-x)^{\lambda} \mathcal{F}_{\rho, \lambda+1}^{\sigma}\left[w(b-x)^{\rho}\right]}{(b-a)^{\lambda+1}}\right] f(x) \\
-\frac{1}{(b-a)^{\lambda+1}}\left[\left(\mathcal{J}_{\rho, \lambda, x-; w}^{\sigma} f\right)(a)+\left(\mathcal{J}_{\rho, \lambda, x+; w}^{\sigma} f\right)(b)\right] \mid \\
\leq\left(\frac{1}{2}\right)^{\frac{1}{q}} \frac{1}{(b-a)^{\lambda+\frac{1}{q}+1}} \\
\times\left\{\left[\left[(b-a)^{2}-(b-x)^{2}\right]\left|f^{\prime}(a)\right|^{q}+(x-a)^{2}\left|f^{\prime}(b)\right|^{q}\right]^{\frac{1}{q}}\right. \\
\times(x-a)^{\lambda+\frac{1}{p}} \mathcal{F}_{\rho, \lambda+1}^{\sigma^{*}}\left[|w|(x-a)^{\rho}\right] \\
+\left[(b-x)^{2}\left|f^{\prime}(a)\right|^{q}+\left[(b-a)^{2}-(x-a)^{2}\right]\left|f^{\prime}(b)\right|^{q}\right]^{\frac{1}{q}} \\
\left.\times(b-x)^{\lambda+\frac{1}{p}} \mathcal{F}_{\rho, \lambda+1}^{\sigma^{*}}\left[|w|(b-x)^{\rho}\right]\right\} .
\end{gathered}
$$

Theorem 4. Let $\varphi: I \longrightarrow A$ be a continuous function. Suppose $A \subseteq \mathbb{R}$ be an open $m$-invex subset with respect to $\eta: A \times A \times(0,1] \longrightarrow \mathbb{R}$ for any fixed $s, m \in(0,1]$ and let $\eta(\varphi(b), \varphi(a), m) \neq 0$. Assume that $f: A \longrightarrow \mathbb{R}$ is a differentiable function on $A^{\circ}$. If $\left|f^{\prime}\right|^{q}$ is generalized $(s, m, \varphi)$-preinvex function on $[m \varphi(a), m \varphi(a)+\eta(\varphi(b), \varphi(a), m)], q \geq 1$, then the following inequality for generalized fractional integral operators holds:

$$
\begin{gathered}
\left|I_{f, \eta, \varphi}(x ; \lambda, \rho, w, m, a, b)\right| \leq\left(\mathcal{F}_{\rho, \lambda+1}^{\sigma_{1}}\left[|w|(x-m \varphi(a))^{\rho}\right]\right)^{1-\frac{1}{q}} \\
\times\left[m\left|f^{\prime}(\varphi(a))\right|^{q} \mathcal{F}_{\rho, \lambda+1}^{\sigma_{2}}\left[|w| \eta^{\rho}(\varphi(b), \varphi(a), m)\right]+\left|f^{\prime}(\varphi(b))\right|^{q} \mathcal{F}_{\rho, \lambda+1}^{\sigma_{3}}\left[|w|(x-m \varphi(a))^{\rho}\right]\right]^{\frac{1}{q}} \\
+\left(\mathcal{F}_{\rho, \lambda+1}^{\sigma_{4}}\left[|w|(m \varphi(a)+\eta(\varphi(b), \varphi(a), m)-x)^{\rho}\right]\right)^{1-\frac{1}{q}} \\
\times\left[m\left|f^{\prime}(\varphi(a))\right|^{q} \mathcal{F}_{\rho, \lambda+1}^{\sigma_{5}}\left[|w|(m \varphi(a)+\eta(\varphi(b), \varphi(a), m)-x)^{\rho}\right]\right. \\
\left.+\left|f^{\prime}(\varphi(b))\right|^{q} \mathcal{F}_{\rho, \lambda+1}^{\sigma_{6}}\left[|w| \eta^{\rho}(\varphi(b), \varphi(a), m)\right]\right]^{\frac{1}{q}}
\end{gathered}
$$

where $\lambda, \rho>0, w \in \mathbb{R}, k=0,1,2, \ldots, \beta(x ; a, b)$ is incompleted beta function and

$$
\begin{gathered}
\sigma_{1}(k)=\sigma(k)\left(\frac{x-m \varphi(a)}{\eta(\varphi(b), \varphi(a), m)}\right) \frac{1}{\rho k+1} ; \\
\sigma_{2}(k)=\sigma(k) \beta\left(\frac{x-m \varphi(a)}{\eta(\varphi(b), \varphi(a), m)} ; \lambda q+\rho k+1, s+1\right) ; \\
\sigma_{3}(k)=\sigma(k)\left(\frac{x-m \varphi(a)}{\eta(\varphi(b), \varphi(a), m)}\right)^{\lambda q+s+1} \frac{1}{\lambda q+\rho k+s+1} ; \\
\sigma_{4}(k)=\sigma(k)\left(\frac{m \varphi(a)+\eta(\varphi(b), \varphi(a), m)-x}{\eta(\varphi(b), \varphi(a), m)}\right) \frac{1}{\rho k+1} ;
\end{gathered}
$$




$$
\begin{gathered}
\sigma_{5}(k)=\sigma(k)\left(\frac{m \varphi(a)+\eta(\varphi(b), \varphi(a), m)-x}{\eta(\varphi(b), \varphi(a), m)}\right)^{\lambda q+s+1} \frac{1}{\lambda q+\rho k+s+1} \\
\sigma_{6}(k)=\sigma(k) \beta\left(\frac{m \varphi(a)+\eta(\varphi(b), \varphi(a), m)-x}{\eta(\varphi(b), \varphi(a), m)} ; \lambda q+\rho k+1, s+1\right) .
\end{gathered}
$$

Proof. Suppose that $q \geq 1$. From Lemma 1, generalized $(s, m, \varphi)$-preinvexity of $\left|f^{\prime}\right|^{q}$, the well-known power mean inequality and properties of the modulus, we have

$$
\begin{aligned}
& \left|I_{f, \eta, \varphi}(x ; \lambda, \rho, w, m, a, b)\right| \\
& \leq \int_{0}^{\frac{x-m \varphi(a)}{\eta(\varphi(b), \varphi(a), m)}} t^{\lambda} \mathcal{F}_{\rho, \lambda+1}^{\sigma}\left[|w| \eta^{\rho}(\varphi(b), \varphi(a), m) t^{\rho}\right]\left|f^{\prime}(m \varphi(a)+t \eta(\varphi(b), \varphi(a), m))\right| d t \\
& +\int_{\frac{x-m \varphi(a)}{\eta(\varphi(b), \varphi(a), m)}}^{1}|1-t|^{\lambda} \mathcal{F}_{\rho, \lambda+1}^{\sigma}\left[|w| \eta^{\rho}(\varphi(b), \varphi(a), m)(1-t)^{\rho}\right] \\
& \times\left|f^{\prime}(m \varphi(a)+t \eta(\varphi(b), \varphi(a), m))\right| d t \\
& \leq\left(\int_{0}^{\frac{x-m \varphi(a)}{\eta(\varphi(b), \varphi(a), m)}} \mathcal{F}_{\rho, \lambda+1}^{\sigma}\left[|w| \eta^{\rho}(\varphi(b), \varphi(a), m) t^{\rho}\right] d t\right)^{1-\frac{1}{q}} \\
& \times\left(\int_{0}^{\frac{x-m \varphi(a)}{\eta(\varphi(b), \varphi(a), m)}} t^{\lambda q} \mathcal{F}_{\rho, \lambda+1}^{\sigma}\left[|w| \eta^{\rho}(\varphi(b), \varphi(a), m) t^{\rho}\right]\left|f^{\prime}(m \varphi(a)+t \eta(\varphi(b), \varphi(a), m))\right|^{q} d t\right)^{\frac{1}{q}} \\
& +\left(\int_{\frac{x-m \varphi(a)}{\eta(\varphi(b), \varphi(a), m)}}^{1} \mathcal{F}_{\rho, \lambda+1}^{\sigma}\left[|w| \eta^{\rho}(\varphi(b), \varphi(a), m)(1-t)^{\rho}\right] d t\right)^{1-\frac{1}{q}} \\
& \times\left(\int_{\frac{x-m \varphi(a)}{\eta(\varphi(b), \varphi(a), m)}}^{1}(1-t)^{\lambda q} \mathcal{F}_{\rho, \lambda+1}^{\sigma}\left[|w| \eta^{\rho}(\varphi(b), \varphi(a), m)(1-t)^{\rho}\right]\left|f^{\prime}(m \varphi(a)+t \eta(\varphi(b), \varphi(a), m))\right|^{q} d t\right)^{\frac{1}{q}} \\
& \leq\left(\sum_{k=0}^{+\infty} \frac{\sigma(k)|w|^{k} \eta^{\rho k}(\varphi(b), \varphi(a), m)}{\Gamma(\lambda+\rho k+1)} \int_{0}^{\frac{x-m \varphi(a)}{\eta(\varphi(b), \varphi(a), m)}} t^{\rho k} d t\right)^{1-\frac{1}{q}} \\
& \times\left[\sum_{k=0}^{+\infty} \frac{\sigma(k)|w|^{k} \eta^{\rho k}(\varphi(b), \varphi(a), m)}{\Gamma(\lambda+\rho k+1)}\right. \\
& \left.\times \int_{0}^{\frac{x-m \varphi(a)}{\eta(\varphi(b), \varphi(a), m)}} t^{\lambda q+\rho k}\left[m(1-t)^{s}\left|f^{\prime}(\varphi(a))\right|^{q}+t^{s}\left|f^{\prime}(\varphi(b))\right|^{q}\right] d t\right]^{\frac{1}{q}} \\
& +\left(\sum_{k=0}^{+\infty} \frac{\sigma(k)|w|^{k} \eta^{\rho k}(\varphi(b), \varphi(a), m)}{\Gamma(\lambda+\rho k+1)} \int_{\frac{x-m \varphi(a)}{\eta(\varphi(b), \varphi(a), m)}}^{1}(1-t)^{\rho k} d t\right)^{1-\frac{1}{q}} \\
& \times\left[\sum_{k=0}^{+\infty} \frac{\sigma(k)|w|^{k} \eta^{\rho k}(\varphi(b), \varphi(a), m)}{\Gamma(\lambda+\rho k+1)}\right. \\
& \left.\times \int_{\frac{x-m \varphi(a)}{\eta(\varphi(b), \varphi(a), m)}}^{1}(1-t)^{\lambda q+\rho k}\left[m(1-t)^{s}\left|f^{\prime}(\varphi(a))\right|^{q}+t^{s}\left|f^{\prime}(\varphi(b))\right|^{q}\right] d t\right]^{\frac{1}{q}} \\
& =\left(\mathcal{F}_{\rho, \lambda+1}^{\sigma_{1}}\left[|w|(x-m \varphi(a))^{\rho}\right]\right)^{1-\frac{1}{q}}
\end{aligned}
$$




$$
\begin{gathered}
\times\left[m\left|f^{\prime}(\varphi(a))\right|{ }^{q} \mathcal{F}_{\rho, \lambda+1}^{\sigma_{2}}\left[|w| \eta^{\rho}(\varphi(b), \varphi(a), m)\right]+\left|f^{\prime}(\varphi(b))\right|^{q} \mathcal{F}_{\rho, \lambda+1}^{\sigma_{3}}\left[|w|(x-m \varphi(a))^{\rho}\right]\right]^{\frac{1}{q}} \\
+\left(\mathcal{F}_{\rho, \lambda+1}^{\sigma_{4}}\left[|w|(m \varphi(a)+\eta(\varphi(b), \varphi(a), m)-x)^{\rho}\right]\right)^{1-\frac{1}{q}} \\
\times\left[m\left|f^{\prime}(\varphi(a))\right|^{q} \mathcal{F}_{\rho, \lambda+1}^{\sigma_{5}}\left[|w|(m \varphi(a)+\eta(\varphi(b), \varphi(a), m)-x)^{\rho}\right]\right. \\
\left.+\left|f^{\prime}(\varphi(b))\right|^{q} \mathcal{F}_{\rho, \lambda+1}^{\sigma_{6}}\left[|w| \eta^{\rho}(\varphi(b), \varphi(a), m)\right]\right]^{\frac{1}{q}}
\end{gathered}
$$

Corollary 4. Under the same conditions as in Theorem 4, if we choose $m=s=$ $1, \eta(\varphi(b), \varphi(a), m)=\varphi(b)-m \varphi(a)$ and $\varphi(x)=x$, we get

$$
\begin{gathered}
\mid\left[\frac{(x-a)^{\lambda} \mathcal{F}_{\rho, \lambda+1}^{\sigma}\left[w(x-a)^{\rho}\right]+(b-x)^{\lambda} \mathcal{F}_{\rho, \lambda+1}^{\sigma}\left[w(b-x)^{\rho}\right]}{(b-a)^{\lambda+1}}\right] f(x) \\
-\frac{1}{(b-a)^{\lambda+1}}\left[\left(\mathcal{J}_{\rho, \lambda, x-; w}^{\sigma} f\right)(a)+\left(\mathcal{J}_{\rho, \lambda, x+; w}^{\sigma} f\right)(b)\right] \mid \\
\leq\left(\mathcal{F}_{\rho, \lambda+1}^{\sigma_{1}^{*}}\left[|w|(x-a)^{\rho}\right]\right)^{1-\frac{1}{q}} \\
\times\left[\left|f^{\prime}(a)\right|^{q} \mathcal{F}_{\rho, \lambda+1}^{\sigma_{2}^{*}}\left[|w|(b-a)^{\rho}\right]+\left|f^{\prime}(b)\right|^{q} \mathcal{F}_{\rho, \lambda+1}^{\sigma_{3}^{*}}\left[|w|(x-a)^{\rho}\right]\right]^{\frac{1}{q}} \\
+\left(\mathcal{F}_{\rho, \lambda+1}^{\sigma_{4}^{*}}\left[|w|(b-x)^{\rho}\right]\right)^{1-\frac{1}{q}} \\
\times\left[\left|f^{\prime}(a)\right|^{q} \mathcal{F}_{\rho, \lambda+1}^{\sigma_{5}^{*}}\left[|w|(b-x)^{\rho}\right]+\left|f^{\prime}(b)\right|^{q} \mathcal{F}_{\rho, \lambda+1}^{\sigma_{6}^{*}}\left[|w|(b-a)^{\rho}\right]\right]^{\frac{1}{q}}
\end{gathered}
$$

where

$$
\begin{gathered}
\sigma_{1}^{*}(k)=\sigma(k)\left(\frac{x-a}{b-a}\right) \frac{1}{\rho k+1} ; \\
\sigma_{2}^{*}(k)=\sigma(k) \beta\left(\frac{x-a}{b-a} ; \lambda q+\rho k+1,2\right) ; \\
\sigma_{3}^{*}(k)=\sigma(k)\left(\frac{x-a}{b-a}\right)^{\lambda q+2} \frac{1}{\lambda q+\rho k+2} ; \\
\sigma_{4}^{*}(k)=\sigma(k)\left(\frac{b-x}{b-a}\right) \frac{1}{\rho k+1} ; \\
\sigma_{5}^{*}(k)=\sigma(k)\left(\frac{b-x}{b-a}\right)^{\lambda q+2} \frac{1}{\lambda q+\rho k+2} ; \\
\sigma_{6}^{*}(k)=\sigma(k) \beta\left(\frac{b-x}{b-a} ; \lambda q+\rho k+1,2\right) .
\end{gathered}
$$

Corollary 5. If we choose $\sigma(0)=1, w=0$ in Corollary 4, the inequality (2.9) reduces to inequality (2.4) of (see [1] Theorem 2.3). 


\section{Applications to special means}

In the following we give certain generalizations of some notions for a positive valued function of a positive variable.

Definition 7. (see [39]) A function $M: \mathbb{R}_{+}^{2} \longrightarrow \mathbb{R}_{+}$, is called a Mean function if it has the following properties:

(1) Homogeneity: $M(a x, a y)=a M(x, y)$, for all $a>0$,

(2) Symmetry: $M(x, y)=M(y, x)$,

(3) Reflexivity: $M(x, x)=x$,

(4) Monotonicity: If $x \leq x^{\prime}$ and $y \leq y^{\prime}$, then $M(x, y) \leq M\left(x^{\prime}, y^{\prime}\right)$,

(5) Internality: $\min \{x, y\} \leq M(x, y) \leq \max \{x, y\}$.

We consider some means for arbitrary positive real numbers $\alpha, \beta(\alpha \neq \beta)$.

(1) The arithmetic mean:

$$
A:=A(\alpha, \beta)=\frac{\alpha+\beta}{2}
$$

(2) The geometric mean:

$$
G:=G(\alpha, \beta)=\sqrt{\alpha \beta}
$$

(3) The harmonic mean:

$$
H:=H(\alpha, \beta)=\frac{2}{\frac{1}{\alpha}+\frac{1}{\beta}}
$$

(4) The power mean:

$$
P_{r}:=P_{r}(\alpha, \beta)=\left(\frac{\alpha^{r}+\beta^{r}}{2}\right)^{\frac{1}{r}}, r \geq 1 .
$$

(5) The identric mean:

$$
I:=I(\alpha, \beta)= \begin{cases}\frac{1}{e}\left(\frac{\beta^{\beta}}{\alpha^{\alpha}}\right), & \alpha \neq \beta \\ \alpha, & \alpha=\beta .\end{cases}
$$

(6) The logarithmic mean:

$$
L:=L(\alpha, \beta)=\frac{\beta-\alpha}{\ln (\beta)-\ln (\alpha)} .
$$

(7) The generalized log-mean:

$$
L_{p}:=L_{p}(\alpha, \beta)=\left[\frac{\beta^{p+1}-\alpha^{p+1}}{(p+1)(\beta-\alpha)}\right]^{\frac{1}{p}} ; p \in \mathbb{R} \backslash\{-1,0\} .
$$

(8) The weighted $p$-power mean:

$$
M_{p}\left(\begin{array}{cccc}
\alpha_{1}, & \alpha_{2}, & \cdots & , \alpha_{n} \\
u_{1}, & u_{2}, & \cdots & , u_{n}
\end{array}\right)=\left(\sum_{i=1}^{n} \alpha_{i} u_{i}^{p}\right)^{\frac{1}{p}}
$$

where $0 \leq \alpha_{i} \leq 1, u_{i}>0(i=1,2, \ldots, n)$ with $\sum_{i=1}^{n} \alpha_{i}=1$. 
It is well known that $L_{p}$ is monotonic nondecreasing over $p \in \mathbb{R}$ with $L_{-1}:=L$ and $L_{0}:=I$. In particular, we have the following inequality $H \leq G \leq L \leq I \leq A$. Now, let $a$ and $b$ be positive real numbers such that $a<b$. Consider the function $M:=M(\varphi(a), \varphi(b)):[\varphi(a), \varphi(a)+\eta(\varphi(b), \varphi(a))] \times[\varphi(a), \varphi(a)+\eta(\varphi(b), \varphi(a))] \longrightarrow$ $\mathbb{R}_{+}$, which is one of the above mentioned means and $\varphi: I \longrightarrow A$ be a continuous function, therefore one can obtain various inequalities using the results of Section 2 for these means as follows: Replace $\eta(\varphi(y), \varphi(x), m)$ with $\eta(\varphi(y), \varphi(x))$ and setting $\eta(\varphi(a), \varphi(b))=M(\varphi(a), \varphi(b))$ for value $m=1$ in (2.4), (2.6) and (2.8), one can obtain the following interesting inequalities involving means:

$$
\begin{aligned}
& \left|I_{f, M(\cdot, \cdot), \varphi}(x ; \lambda, \rho, w, 1, a, b)\right|=\left\lfloor\left[\frac{(x-\varphi(a))^{\lambda} \mathcal{F}_{\rho, \lambda+1}^{\sigma}\left[w(x-\varphi(a))^{\rho}\right]}{M^{\lambda+1}(\varphi(a), \varphi(b))}\right.\right. \\
& \left.+\frac{(\varphi(a)+M(\varphi(a), \varphi(b))-x)^{\lambda} \mathcal{F}_{\rho, \lambda+1}^{\sigma}\left[w(\varphi(a)+M(\varphi(a), \varphi(b))-x)^{\rho}\right]}{M^{\lambda+1}(\varphi(a), \varphi(b))}\right] f(x) \\
& -\frac{1}{M^{\lambda+1}(\varphi(a), \varphi(b))} \\
& \times\left[\left(\mathcal{J}_{\rho, \lambda, x-; w}^{\sigma} f\right)(\varphi(a))+\left(\mathcal{J}_{\rho, \lambda, x+; w}^{\sigma} f\right)(\varphi(a)+M(\varphi(a), \varphi(b)))\right] \mid \\
& \leq\left[\frac{(\varphi(a)+M(\varphi(a), \varphi(b))-x)^{\lambda+s+1}}{M^{\lambda+s+1}(\varphi(a), \varphi(b))} \mathcal{F}_{\rho, \lambda+1}^{\sigma_{2}}\left[|w|(\varphi(a)+M(\varphi(a), \varphi(b))-x)^{\rho}\right]\right. \\
& \left.+\mathcal{F}_{\rho, \lambda+1}^{\sigma_{1}}\left[|w| M^{\rho}(\varphi(a), \varphi(b))\right]\right]\left|f^{\prime}(\varphi(a))\right| \\
& +\left[\frac{(x-\varphi(a))^{\lambda+s+1}}{M^{\lambda+s+1}(\varphi(a), \varphi(b))} \mathcal{F}_{\rho, \lambda+1}^{\sigma_{2}}\left[|w|(x-\varphi(a))^{\rho}\right]\right. \\
& \left.+\mathcal{F}_{\rho, \lambda+1}^{\sigma_{3}}\left[|w| M^{\rho}(\varphi(a), \varphi(b))\right]\right]\left|f^{\prime}(\varphi(b))\right|, \\
& \left|I_{f, M(\cdot, \cdot), \varphi}(x ; \lambda, \rho, w, 1, a, b)\right| \leq \frac{1}{(s+1)^{\frac{1}{q}}} \frac{1}{M^{\lambda+\frac{s}{q}+1}(\varphi(a), \varphi(b))} \\
& \times\left\{\left[\left[M^{s+1}(\varphi(a), \varphi(b))-(\varphi(a)+M(\varphi(a), \varphi(b))-x)^{s+1}\right]\left|f^{\prime}(\varphi(a))\right|^{q}\right.\right. \\
& \left.+(x-\varphi(a))^{s+1}\left|f^{\prime}(\varphi(b))\right|^{q}\right]^{\frac{1}{q}}(x-\varphi(a))^{\lambda+\frac{1}{p}} \mathcal{F}_{\rho, \lambda+1}^{\sigma^{*}}\left[|w|(x-\varphi(a))^{\rho}\right] \\
& +\left[\left[(\varphi(a)+M(\varphi(a), \varphi(b))-x)^{s+1}\right]\left|f^{\prime}(\varphi(a))\right|^{q}\right. \\
& \left.+\left[M^{s+1}(\varphi(a), \varphi(b))-(x-\varphi(a))^{s+1}\right]\left|f^{\prime}(\varphi(b))\right|^{q}\right]^{\frac{1}{q}} \\
& \left.\times(\varphi(a)+M(\varphi(a), \varphi(b))-x)^{\lambda+\frac{1}{p}} \mathcal{F}_{\rho, \lambda+1}^{\sigma^{*}}\left[|w|(\varphi(a)+M(\varphi(a), \varphi(b))-x)^{\rho}\right]\right\}, \\
& \left|I_{f, M(\cdot, \cdot), \varphi}(x ; \lambda, \rho, w, 1, a, b)\right| \leq\left(\mathcal{F}_{\rho, \lambda+1}^{\sigma_{1}}\left[|w|(x-\varphi(a))^{\rho}\right]\right)^{1-\frac{1}{q}}
\end{aligned}
$$




$$
\begin{gathered}
\times\left[\left|f^{\prime}(\varphi(a))\right|^{q} \mathcal{F}_{\rho, \lambda+1}^{\sigma_{2}}\left[|w| M^{\rho}(\varphi(a), \varphi(b))\right]+\left|f^{\prime}(\varphi(b))\right|^{q} \mathcal{F}_{\rho, \lambda+1}^{\sigma_{3}}\left[|w|(x-\varphi(a))^{\rho}\right]\right]^{\frac{1}{q}} \\
+\left(\mathcal{F}_{\rho, \lambda+1}^{\sigma_{4}}\left[|w|(\varphi(a)+M(\varphi(a), \varphi(b))-x)^{\rho}\right]\right)^{1-\frac{1}{q}} \\
\times\left[\left|f^{\prime}(\varphi(a))\right|^{q} \mathcal{F}_{\rho, \lambda+1}^{\sigma_{5}}\left[|w|(\varphi(a)+M(\varphi(a), \varphi(b))-x)^{\rho}\right]\right. \\
\left.+\left|f^{\prime}(\varphi(b))\right|^{q} \mathcal{F}_{\rho, \lambda+1}^{\sigma_{6}}\left[|w| M^{\rho}(\varphi(a), \varphi(b))\right]\right]^{\frac{1}{q}} .
\end{gathered}
$$

Letting $M(\varphi(a), \varphi(b))=A, G, H, P_{r}, I, L, L_{p}, M_{p}$ in (3.1), (3.2) and (3.3), we get the inequalities involving means for a particular choice of a differentiable generalized $(s, 1, \varphi)$-preinvex functions $f$. The details are left to the interested reader.

\section{Conclusions}

In the present paper, the notion of generalized $(s, m, \varphi)$-preinvex function was applied to established some new generalizations of Ostrowski type inequalities via fractional integral operators. These results not only extended the results appeared in the literature (see [1]) but also provided new estimates on these type. Some applications to special means are obtained.

Motivated by this new interesting class of generalized $(s, m, \varphi)$-preinvex functions we can indeed see to be vital for fellow researchers and scientists working in the same domain.

We conclude that our methods considered here may be a stimulant for further investigations concerning Hermite-Hadamard and Ostrowski type integral inequalities for various kinds of preinvex functions involving classical integrals, RiemannLiouville fractional integrals, $k$-fractional integrals, local fractional integrals, fractional integral operators, $q$-calculus, $(p, q)$-calculus, time scale calculus and conformable fractional integrals.

\section{REFERENCES}

[1] Ç. Yildiz, M. E. Özdemir and M. Z. Sarikaya, New generalazations of Ostrowski-like type inequalities for fractional integrals, Kyungpook Math. J., 56, (2016), 161-172.

[2] A. Kashuri and R. Liko, Ostrowski type fractional integral inequalities for generalized ( $s, m, \varphi)$-preinvex functions, Aust. J. Math. Anal. Appl., 13 (1) (2016), Article 16, 1-11.

[3] M. Alomari, M. Darus, S. S. Dragomir and P. Cerone, Ostrowski type inequalities for functions whose derivatives are $s$-convex in the second sense, Appl. Math. Lett., 23, (2010), 1071-1076.

[4] R. P. Agarwal, M. J. Luo and R. K. Raina, On Ostrowski type inequalities, Fasc. Math., 204, (2016), 5-27.

[5] S. S. Dragomir, On the Ostrowski's integral inequality for mappings with bounded variation and applications, Math. Ineq. \& Appl., 1, (2) (1998).

[6] S. S. Dragomir, The Ostrowski integral inequality for Lipschitzian mappings and applications, Comput. Math. Appl., 38, (1999), 33-37.

[7] Z. Liu, Some Ostrowski-Grüss type inequalities and applications, Comput. Math. Appl., 53, (2007), 73-79.

[8] M. E. Özdemir, H. Kavurmac and E. Set, Ostrowski's type inequalities for $(\alpha, m)$-convex functions, Kyungpook Math. J., 50, (2010), 371-378.

[9] B. G. Pachpatte, On an inequality of Ostrowski type in three independent variables, $J$. Math. Anal. Appl., 249, (2000), 583-591. 
[10] B. G. Pachpatte, On a new Ostrowski type inequality in two independent variables, Tamkang J. Math., 32, (1) (2001), 45-49.

[11] A. Rafiq, N. A. Mir and F. Ahmad, Weighted Čebyšev-Ostrowski type inequalities, Applied Math. Mechanics (English Edition), 28, (7) (2007), 901-906.

[12] N. Ujević, Sharp inequalities of Simpson type and Ostrowski type, Comput. Math. Appl., 48, (2004), 145-151.

[13] L. Zhongxue, On sharp inequalities of Simpson type and Ostrowski type in two independent variables, Comput. Math. Appl., 56, (2008), 2043-2047.

[14] M. Adil Khan, T. Ali, S. S. Dragomir, M. Z. Sarikaya, Hermite-Hadamard type inequalities for conformable fractional integrals, Revista de la Real Academia de Ciencias Exactas, Fsicas y Naturales. Serie A. Matemàticas, (2017). doi:10.1007/s13398-017-0408-5.

[15] T. Abdeljawad, On conformable fractional calculus, J. Comput. Appl. Math., 279, (2015), 57-66.

[16] Z. Dahmani, New inequalities in fractional integrals, Int. J. Nonlinear Sci., 9, (4) (2010), 493-497.

[17] Z. Dahmani, L. Tabharit and S. Taf, Some fractional integral inequalities, Nonlinear. Sci. Lett. A, 1, (2) (2010), 155-160.

[18] Z. Dahmani, On Minkowski and Hermite-Hadamard integral inequalities via fractional integration, Ann. Funct. Anal., 1, (1) (2010), 51-58.

[19] Z. Dahmani, L. Tabharit and S. Taf, New generalizations of Grüss inequality using RiemannLiouville fractional integrals, Bull. Math. Anal. Appl., 2, (3) (2010), 93-99.

[20] U. N. Katugampola, A new approach to generalized fractional derivatives, Bulletin Math. Anal. Appl., 6, (4) (2014), 1-15.

[21] R. Khalil, M. Al Horani, A. Yousef and M. Sababheh, A new definition of fractional derivative, J. Comput. Appl. Math., 264, (2014), 65-70.

[22] S. D. Purohit and S. L. Kalla, Certain inequalities related to the Chebyshev's functional involving Erdelyi-Kober operators, Scientia Series A: Math. Sci., 25, (2014), 53-63.

[23] R. K. Raina, On generalized Wright's hypergeometric functions and fractional calculus operators, East Asian Math. J., 21, (2) (2005), 191-203.

[24] M. Z. Sarikaya, On the Ostrowski type integral inequality, Acta Math. Univ. Comenianae, 79, (1) (2010), 129-134.

[25] E. Set, A. Gözpnar and J. Choi, Hermite-Hadamard type inequalities for twice differentiable m-convex functions via conformable fractional integrals, Far East J. Math. Sci., 101, (4) (2017), 873-891.

[26] T. S. Du, J. G. Liao and Y. J. Li, Properties and integral inequalities of Hadamard-Simpson type for the generalized $(s, m)$-preinvex functions, J. Nonlinear Sci. Appl., 9, (2016), 31123126.

[27] H. Hudzik and L. Maligranda, Some remarks on s-convex functions, Aequationes Math., 48, (1994), 100-111.

[28] M. Ahmadmir and R. Ullah, Some inequalities of Ostrowski and Grüss type for triple integrals on time scales, Tamkang J. Math., 42, (4) (2011), 415-426.

[29] S. S. Dragomir and S. Wang, A new inequality of Ostrowski's type in $L_{1}$-norm and applications to some special means and to some numerical quadrature rules, Tamkang J. Math., 28, (1997), 239-244.

[30] S. S. Dragomir and S. Wang, An inequality of Ostrowski-Grüss type and its applications to the estimation of error bounds for some special means and for some numerical quadrature rules, Comput. Math. Appl., 13, (11) (1997), 15-20.

[31] Z. Liu, Some companions of an Ostrowski type inequality and applications, J. Inequal. in Pure and Appl. Math, 10, (2) (2009), Art. 52, 12 pp.

[32] D. S. Mitrinovic, J. E. Pečarić and A. M. Fink, Classical and new inequalities in analysis, Kluwer Academic Publishers, Dordrecht, (1993).

[33] T. Antczak, Mean value in invexity analysis, Nonlinear Anal., 60, (2005), 1473-1484.

[34] X. M. Yang, X. Q. Yang and K. L. Teo, Generalized invexity and generalized invariant monotonicity, J. Optim. Theory Appl., 117, (2003), 607-625.

[35] R. Pini, Invexity and generalized convexity, Optimization, 22, (1991), 513-525. 
[36] W. Liu, W. Wen and J. Park, Hermite-Hadamard type inequalities for MT-convex functions via classical integrals and fractional integrals, J. Nonlinear Sci. Appl., 9, (2016), 766-777.

[37] W. Liu, W. Wen and J. Park, Ostrowski type fractional integral inequalities for MT-convex functions, Miskolc Math. Notes, 16, (1) (2015), 249-256.

[38] M. Tunç, Ostrowski type inequalities for functions whose derivatives are MT-convex, $J$. Comput. Anal. Appl., 17, (4) (2014), 691-696.

[39] P. S. Bullen, Handbook of Means and Their Inequalities, Kluwer Academic Publishers, Dordrecht, (2003).

Department of Mathematics, Faculty of Technical Science, University "Ismail Qemali", Vlora, Albania

E-mail address: artionkashuri@gmail.com

Department of Mathematics, Faculty of Technical Science, University "Ismail Qemali", Vlora, Albania

E-mail address: rozanaliko86@gmail.com 